\title{
ON A GENERAL INEQUALITY RELATED TO THE GENERALIZED-EULER-CONSTANT FUNCTION
}

\author{
HAN-QI TANG AND AI-Min XU
}

Abstract. Let $\gamma(z)$ be the generalized-Euler-constant function. In this paper, we establish a general inequality related to $\gamma(z)$, which contains a result due to Chen and Han as a special case. We also obtain an inequality for the generalized Somos recurrence constant, using its relation with the generalized-Euler-constant function.

Mathematics subject classification (2010): 26D15. function.

Keywords and phrases: Generalized Somos recurrence constant, inequality, generalized-Euler-constant

\section{REFERENCES}

[1] C.P. CHEn, New asymptotic expasions related to Somos' quadratic recurrence constant, C. R. Acad. Sci. Paris, Ser. I. 351(2013), 9-12.

[2] C.P. Chen, X.F. HAn, On Somos' quadratic recurrence constant, J. Number Theory 166(2016), $31-40$.

[3] S.R. FInCH, Mathematical Constants, Cambridge University Press, Cambridge, England, 2003.

[4] J. Guillera, J. Sondow, Double integrals and infinite products for some classical constants via analytic continuations of Lerch's transcendent, Ramanujan J. 16(2008), 247-270.

[5] M.D. HirschHorn, A note on Somos' quadratic recurrence constant, J. Number Theory 131(2011), 2061-2063.

[6] V. LAMPRET, Approximation of Sondow's generalized-Euler-constant function on the interval [-1,1], Ann. Univ. Ferrara 56(2010), 65-76.

[7] D. Lu, Z. Song, Some new continued fraction estimates of the Somos' quadratic recurrence constant, J. Number Theory 155(2015), 36-45.

[8] X.S. MA, C.P. CHEN, Inequalities and asymptotic expansions related to the generalized Somos quadratic recurrence constant, J. Inequal. Appl. 2018(2018), Article 147.

[9] C. MoRTICI, Estimating the Somos' quadratic recurrence constant, J. Number Theory 130(2010), 2650-2657.

[10] G. NEMES, On the coefficients of an asymptotic expansion related to Somos' quadratic recurrence constant, Appl. Anal. Discrete Math. 5(2011), 60-66.

[11] K.H. PILEhrood, T.H. PILEHROOD, Arithmetical properties of some series with logarithmic coefficients, Math. Z. 255(2007), 117-131.

[12] K.H. Pilehrood, T.H. Pilehrood, Vacca-type series for values of the generalized Euler constant constant function and its derivative, J. Integer Seq. 13(2010), Article ID 10.7.3.

[13] S. Ramanujan, G.H. Hardy, P.V.S. Aiyar, B.M. Wilson (eds.), Collected Papers of Srinivasa Ramanujan, Amer. Math. Soc. Providence, 2000.

[14] M. Somos, Several constants related to quadratic recurrences, unpublished note, 1999.

[15] J. Sondow, Double integrals for Euler's constant and $\ln (4 / \pi)$ and an analog of Hadjicostas's formula, Amer. Math. Monthly, 112(2005), 61-65.

[16] J. Sondow, New Vacca-type rational series for Euler's constant and its “alternating” analog in $\ln (4 / \pi)$, In: Additive Number Theory, pp. 331-340, Springer, New York, 2010.

[17] J. SONDOW, P. HADJICOSTAS, The generalized-Euler-constant function $\gamma(z)$ and a generalization of Somos's quadratic recurrence constant, J. Math. Anal. Appl. 332(2007), 292-314. 
[18] E.W. WeIsstein, Somos's quadratic recurrence constant, MathWorld-A Wolfram Web Resource, Published electronically at http://mathworld.wolfram. com/SomossQuadraticRecurrenceConstant.html.

[19] A. XU, Asymptotic expansion related to the generalized Somos recurrence constant, Int. J. Number Theory, 15(2019), 2043-2055.

[20] X. You, D.R. Chen, Improved continued fraction sequence convergent to the Somos' quadratic recurrence constant, J. Math. Anal. Appl. 436(2016), 513-520. 